\title{
Reconstruction of the ion plasma parameters from the current measurements: mathematical tool
}

\author{
E. Séran ${ }^{1}$ \\ ${ }^{1}$ CETP, Observatoire de Saint-Maur, 4, Avenue de Neptune, 94107 Saint-Maur-des-Fossés, Cedex, France
}

Received: 9 April 2002 - Revised: 22 November 2002 - Accepted: 27 November 2002

\begin{abstract}
Instrument d'Analyse du Plasma (IAP) is one of the instruments of the newly prepared ionospheric mission Demeter. This analyser was developed to measure flows of thermal ions at the altitude of $\sim 750 \mathrm{~km}$ and consists of two parts: (i) retarding potential analyser (APR), which is utilised to measure the energy distribution of the ion plasma along the sensor look direction, and (ii) velocity direction analyser (ADV), which is used to measure the arrival angle of the ion flow with respect to the analyser axis. The necessity to obtain quick and precise estimates of the ion plasma parameters has prompted us to revise the existing mathematical tool and to investigate different instrumental limitations, such as (i) finite angular aperture, (ii) grid transparency, (iii) potential depression in the space between the grid wires, (iv) losses of ions during their passage between the entrance diaphragm and the collector. Simple analytical expressions are found to fit the currents, which are measured by the APR and ADV collectors, and show a very good agreement with the numerical solutions. It was proven that the fitting of the current with the model functions gives a possibility to properly resolve even minor ion concentrations and to find the arrival angles of the ion flow in the multi-species plasma. The discussion is illustrated by an analysis of the instrument response in the ionospheric conditions which are predicted by the International Reference Ionosphere (IRI) model.
\end{abstract}

Key words. Ionosphere (plasma convection; instruments and techniques) - Space plasma physics (experimental and mathematical techniques)

\section{Introduction}

The idea to use retarding and drift analysers for diagnostics of the cold ion population is not new. These techniques have grown since the sixties (Knudsen, 1964) and have provided valuable measurements in the ionospheres of Earth (Hanson et al., 1970), Venus (Knudsen et al., 1980) and Mars (Hanson

Correspondence to: E. Séran (seran@ @etp.ipsl.fr) et al., 1977). Nevertheless, the analytical tool which was first proposed by Whipple (1959) to extract the plasma parameters from the current measurements has never been revised from the point of view of the limits of its application and of the accuracy of estimated parameters. The necessity to prepare the data processing of the IAP instrument has prompted us to come back to the sixties with the two-fold aim: (i) to analyse all possible instrumental limitations and (ii) to justify the analytical solutions that fit correctly the current measurements. The assumptions which are made are the following:

1. Ion distribution function is Maxwellian and isotrope, i.e. $T_{\|}=T_{\perp}$

2. Plasma consists of the ion species, $\mathrm{H}^{+}, \mathrm{He}^{+}$and $\mathrm{O}^{+}$, with the concentration of $\sim 10^{8}-10^{11} \mathrm{~m}^{-3}$ and the temperature of $\sim 0.07-0.2 \mathrm{eV}$, bulk and thermal velocities of which are lower than satellite speed;

3. Retarding grids considered as the potential barriers that modify the velocity of input particles in the direction perpendicular to the grid plane and the losses of particles caused by their collisions with the grid wires are introduced through the coefficient of the grid transparency.

We (i) briefly summarize the analysers geometry (Sect. 2); (ii) give the analytical expressions for the current response and compare them with the numerical solution; (iii) discuss the instrumental characteristics, such as the grid transparency, potential depression between the grid wires, angular aperture, effective section of the entrance diaphragm; (iv) analyse the effect of the particle losses on the side walls of the analyser and the contribution of the ions' thermal motion to the current (Sect. 3). 


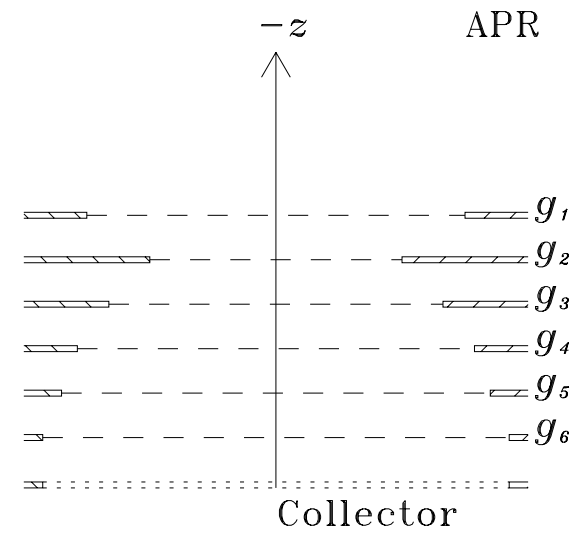

(a)

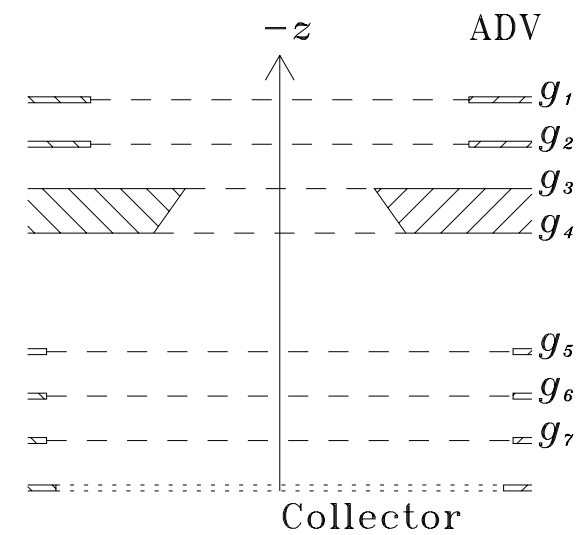

(b)

Fig. 1. Sketches of the APR and ADV analysers. Collectors are shown by dotted lines, grids by slashed lines and the grounded structures by shaded surfaces. Axis $z$ is aligned with satellite speed.

\section{Analysers geometry}

\subsection{APR}

The APR analyser consists of (Fig. 1a) (i) collector of the radius $37 \mathrm{~mm}$, (ii) entrance diaphragm of the radius $r_{d}=20 \mathrm{~mm}$ at the height $h=15 \mathrm{~mm}$ from the collector and (iii) six grids, which are placed parallel to the collector, i.e. perpendicular to the analyser axis $z$. The top grids, $g_{1}$ and $g_{2}$, and the grid $g_{6}$, are maintained at the potential of the satellite structure with the purpose to exclude any perturbations of the ambient plasma caused by the potential variations at the neighbouring grids, $g_{3}, g_{4}$ and $g_{5}$. The next two grids, $g_{3}$ and $g_{4}$, are retarding grids, i.e. positive potential, $\varphi_{g}$, which is applied to them does not allow the ions that flow in the $+z$ direction with the energies lower than $\sim e \varphi_{g}$ to reach the collector. Retarding potential may vary from -2 to $+22 \mathrm{~V}$, i.e. may suppress the ionospheric ions from $\mathrm{H}^{+}$to $\mathrm{Fe}^{+}$. Each grid represents a net of wires that are placed in perpendicular directions, two neighbouring parallel wires are separated by the distance $a \approx 0.5 \mathrm{~mm}$ and a cross section of each wire is a square with the side $\delta \approx 0.03 \mathrm{~mm}$. The potential depression in the space between the grid wires is the function of the grid separation distance, $d$, the grid spacing, $a$, and the wire thickness, $\delta$. In the conditions $\pi \delta / a \ll 1<d / a$ the average potential depression may be written in the following form (see, for example, Hanson et al., 1972)

$\varphi_{g}^{*} \approx \varphi_{g}\left(1-\frac{\kappa a}{2 \pi d^{*}} \ln \left[\frac{a}{\pi \delta}\right]\right)$.

Here, $\kappa$ is the leakage parameter of the square grid with respect to the linear grid and the effective grid separation distance, $d^{*}$ equals $d / 2$ in the configuration with one retarding grid and $\sim d$ in the double-grid configuration. For the APR design with $\kappa \approx 1.72, d=3 \mathrm{~mm}$, the average potential depression is estimated to be $\varphi_{g}^{*} \approx 0.85 \varphi_{g}$ and $\varphi_{g}^{*} \approx 0.92 \varphi_{g}$ for one-grid and double-grid configurations, respectively.

Negative potential, $-12 \mathrm{~V}$, which is applied to the grid $g_{5}$ has a three-fold aim: (i) it cuts the photoelectron current on the collector, (ii) prevents the access of thermal electrons to the collector and (iii) reduces emission of secondary electrons from the collector. Overall, the system of the grids does not change the initial energy of the particle which arrives on the collector.

\subsection{ADV}

The ADV analyser consists of (Fig. 1b) (i) collector of the radius $35.5 \mathrm{~mm}$, (ii) entrance diaphragm with the side of $30 \mathrm{~mm}$ at the height $20 \mathrm{~mm}$ from the collector and (iii) seven grids, which are mounted parallel to the collector. In order to exclude any perturbations of the ambient plasma caused by the potential variations at the grids, $g_{2}$ and $g_{7}$, the external grid, $g_{1}$, and the internal grids, $g_{3}, g_{4}, g_{5}$ and $g_{6}$, are grounded. Positive potential, $+2 \mathrm{~V}$, which may be optionally applied to the grid $g_{2}$ will suppress the ions with $z$-aligned velocity lower than $\sim 2 \cdot 10^{4}\left[m_{i} / m_{H+}\right]^{-0.5} \mathrm{~m} \mathrm{~s}^{-1}$; here, $m_{i}$ and $m_{H+}$ are the masses of the ion species and of hydrogen, respectively. Therefore, all hydrogen and almost all helium will be suppressed by the grid potential, if we assume that (i) bulk velocity of plasma in the satellite frame is determined mainly by the satellite speed, which is aligned with the $z$-axis and is estimated to be $\sim 7.25 \cdot 10^{3} \mathrm{~m} \mathrm{~s}^{-1}$, and that (ii) the thermal speed of the ions at the altitudes of $\sim 750 \mathrm{~km}$ is expected not to exceed the value $\sim 6 \cdot 10^{3}\left[m_{i} / m_{H+}\right]^{-0.5} \mathrm{~m} \mathrm{~s}^{-1}$. The negative potential, $-12 \mathrm{~V}$, at the grid $g_{7}$, nearby with the collector, prevents the collection of the electron and photoelectron currents.

\section{Analysers response on the ion flows}

\subsection{Rough estimate of the current (order of magnitude)}

Ion flows that reach the analyser's collector produce the current which can be roughly estimated as

$J=e S \sum_{i} F_{i}$. 
Here, $e$ is the elementary charge $\left(e \approx 1.6 \cdot 10^{-19} C\right), S$ is the analyser entrance area $\left(1.26 \cdot 10^{-3} \mathrm{~m}^{2}\right.$ for APR and $0.9 \cdot 10^{-3} \mathrm{~m}^{2}$ for ADV), $F_{i}$ is the flux of the ion species $i$. Let us assume that plasma is cold, immobile and consists of only one ion species with the density $n_{i}$. Then the ion flux on the collector is determined by the satellite speed $v_{s c}$, and may be simply written as $F_{i} \approx n_{i} v_{s c}$. The characteristic density of the main ion population, either oxygen (on the dayside) or hydrogen (on the nightside), at the satellite altitude (i.e. $\sim 750 \mathrm{~km}$ ) is predicted to be about $n_{i} \approx 10^{11} \mathrm{~m}^{-3}$. Therefore, the currents which are expected to be collected are $\sim 500 \mathrm{nA}$ for APR and $\sim 460 \mathrm{nA}$ for ADV. Nevertheless, precise calculations of the ion fluxes and the collected currents are complicated by the following effects:

- non-zero temperature of ion population;

- non-zero bulk velocity of ion species in the Earth's frame;

- finite angular aperture of the analyser;

- retarding effect of the grids;

- losses of ions on the grids and on the side parts of the analyser;

- finite value of satellite potential, etc.

All of these issues will be addressed in the following sections.

\subsection{Transparency of the grids}

Before an ion arrives at the collector, it passes a number of grids that are mounted between the analyser entrance and the collector. Hereafter, we assume that an ion which collides with the grid wire is then absorbed and, therefore, does not arrive at the collector. The number of ions that pass the grid is proportional to the ratio of the space surface between the wires and the full section of the entrance diaphragm. If the ion population is cold and the main component of its velocity points along the analyser axis, i.e. perpendicular to the grids, then the grid transparency is estimated to be $(a-\delta)^{2} / a^{2} \approx 0.884$. If the analyser now consists of $n$ grids, then input flux will be reduced by the factor $0.884^{n}$ when it reaches the collector. This factor is estimated to be equal to $\sim 0.48$ and $\sim 0.42$ for APR and ADV analysers, respectively. In the case when the velocity transversal to the analyser axis represents $\sim 10 \%$ of the parallel velocity, the transparencies reduce down to $\sim 0.44$ and $\sim 0.39$ for APR and ADV, respectively.

\subsection{Ion distribution function}

The ion distribution function is assumed to be Maxwellian and isotropic and, therefore, in the plasma frame it can be presented in the form

$f_{i}=f_{o i} \exp \left(-\beta_{i}^{2} \mathbf{v}_{i}^{2}\right)$.
Here, $\beta_{i}=\sqrt{m_{i} / 2 k T}, m_{i}$ and $T_{i}$ are the ion mass and temperature, $k$ is the Boltzmann constant $\left(k=1.38 \cdot 10^{-23} \mathrm{~J} \mathrm{~K}^{-1}\right)$ and $\mathbf{v}_{i}$ is the bulk velocity. The quantity $f_{o i}$ can be expressed in terms of the ion density, $n_{i}$, using the fact that the density is the first moment of the distribution function. Therefore, in a spherical system of coordinates $(v, \theta, \varphi)$ it reads

$$
\begin{aligned}
& n_{i}=\int_{0}^{2 \pi} d \varphi \int_{0}^{\pi} \sin \theta d \theta \int_{0}^{\infty} f v^{2} d v=\frac{\pi^{3 / 2}}{\beta^{3}} f_{o i} \text { and } \\
& f_{o i}=\frac{\beta^{3}}{\pi^{3 / 2}} n_{i}=\left[\frac{m_{i}}{2 \pi k T_{i}}\right]^{3 / 2} n_{i} .
\end{aligned}
$$

To simplify the analytical calculations we assume here that the main component of the ion velocity is parallel to the analyser axis and is determined mainly by the satellite speed. Then the distribution function in the satellite frame reads

$f_{i}=f_{o i} \exp \left(-\beta_{i}^{2}\left(\mathbf{v}-\mathbf{v}_{\|}\right)^{2}\right)$,

with $\mathbf{v}=\{v \cos \theta, \quad v \sin \theta \cos \varphi, \quad v \sin \theta \sin \varphi\}$ and $\mathbf{v}=$ $\left\{v_{\|}, 0,0\right\}$, and can be re-written in the form

$f_{i}=f_{o i} \exp \left(-\beta_{i}^{2} v_{\|}^{2}\right) \exp \left(-\beta_{i}^{2} v^{2}+2 \beta_{i}^{2} \cos \theta v_{\|} v\right)$.

\subsection{Ion flux on the APR collector}

\subsubsection{1-D analytical solution}

Let us assume first that the bulk velocity of ions in the satellite frame is much larger than its thermal velocity. In this case the ion flux on the collector may be regarded as collimated along the analyser axis and, therefore, estimated by evaluating the one-dimensional (1-D) integral of the distribution function (6) along the $v$-axis,

$$
\begin{aligned}
& F_{i}=f_{o i} e^{-\beta_{i}^{2} v_{\|}^{2}} \int_{v_{g}}^{\infty} e^{-\beta_{i}^{2} v^{2}+2 \beta_{i}^{2} v_{\|} v} \\
& v d v=\frac{n_{i} v_{\|}}{2}\left\{1+\Phi\left(\beta_{i}\left(v_{\|}-v_{g}\right)\right)+\frac{e^{-\beta_{i}^{2}\left(v_{\|}-v_{g}\right)^{2}}}{\sqrt{\pi} \beta_{i} v_{\|}}\right\} .
\end{aligned}
$$

Here, $\Phi$ is the error function and $v_{g}$ is the velocity that corresponds to the effective retarding potential $\varphi_{g}^{*}$, i.e. $v_{g}=$ $\sqrt{2 k e \varphi_{g}^{*} / m_{i}}$, and the density in the 1-D case is expressed as $n_{i}=f_{o i} \sqrt{\pi} / \beta_{i}$. Note that the retarding potential is given in the reference of the satellite ground, $\varphi_{s c}$, and consequently the potential which is seen by the ions is $\left(\varphi_{g}^{*}+\varphi_{s c}\right)$. At the ionospheric altitudes of $\sim 750 \mathrm{~km}$ the dominant population is the thermal electrons and, therefore, the potential of the satellite structure is expected to be slightly negative, i.e. to be varied between -0.2 and $-1 \mathrm{~V}$. This produces a preacceleration of ions that enter into the analyser.

In a cold plasma $(\beta \rightarrow \infty)$ the contribution of each ion species in the total flux is a constant and equals $n_{i} v_{\|}$. Therefore, the current-voltage response of the APR collector on each ion species represents a step-function: $J_{i}\left(\varphi_{g}\right)=$ 
$e S n_{i} v_{\|}$, if $0 \leq \varphi_{g}<\frac{m_{i} v_{\|}^{2}}{2 k e}$, and $J_{i}\left(\varphi_{g}\right)=0$, if $\varphi_{g} \geq \frac{m_{i} v_{\|}^{2}}{2 k e}$. For instance, if plasma is immobile, then $v_{\|}=v_{s c}$, and the retarding potential which corresponds to the current cutoff is estimated to be $0.274\left[m_{i} / m_{H+}\right] \mathrm{V}$.

In a warm plasma the analyser response is no longer a stepfunction, but approximately follows the law $0.5 e S n_{i} v_{\|}\{1+$ $\left.\Phi\left(\beta_{i}\left(v_{\|}-v_{g}\right)\right)\right\}$. The effective broadening of the currentvoltage response, i.e. the voltage range, $\Delta \varphi_{g}$, with the lower limit, which corresponds to the departure of the current from the $J_{o i}=e S n_{i} v_{\|}$level, and with the upper limit, which is associated with the current drop down to the value $\sim J_{o i} / 100$, is proportional to the square-root of the ion temperature and parallel velocity, or more precisely, is $\Delta \varphi_{g} \approx \frac{3.6}{e} \sqrt{\frac{2 m_{i} T_{i}}{k}} v_{\|}$. For example, in the oxygen plasma with the temperature of $0.2 \mathrm{eV}$ the broadening is estimated to be $\sim 6.7 \mathrm{~V}$.

\subsubsection{3-D analytical solution}

More precise estimations of the ion flux on the collector may be obtained by taking into account the acceptance angle of analyser and integrating the distribution function in threedimensional (3-D) velocity space inside of cone with the half-angle $\theta^{*}=\operatorname{arctg}\left(r_{d} / h\right)\left(53.1^{\circ}\right.$ for APR). The ion flux on the collector can now be written in the form

$$
\begin{aligned}
& F_{i}=2 \pi f_{o i} e^{-\beta_{i} v_{\|}^{2}} \int_{0}^{\theta^{*}} \sin \theta e^{2 \beta_{i}^{2} v v_{\|} \cos \theta} d \theta \\
& \int_{v_{g}}^{\infty} \exp \left(-\beta_{i}^{2} v^{2}\right) v^{3} d v
\end{aligned}
$$

After integrating, Eq. (8) reads

$$
\begin{aligned}
F_{i}= & \pi f_{o i} \frac{1}{2 \beta_{i}^{4}}\left(K(0)-K\left(\theta_{*}\right)\right) \\
& =F_{o i}\left(K(0)-K\left(\theta_{*}\right)\right),
\end{aligned}
$$

with

$$
F_{o i}=\frac{n_{i}}{2 \beta_{i} \sqrt{\pi}}=n_{i} \sqrt{\frac{k T_{i}}{2 \pi m_{i}}}
$$

and

$$
\begin{aligned}
K(\theta) & =\left(\frac{v_{g}}{v_{\|}}+\cos \theta\right) e^{-\beta_{i}^{2}\left(v_{\|}^{2}-2 v_{g} v_{\|} \cos \theta+v_{g}^{2}\right)+} \\
+ & \frac{\sqrt{\pi}}{2 \beta_{i} v_{\|}} e^{-\beta_{i}^{2} v_{\|}^{2} \sin ^{2} \theta} \\
& \left(1+\Phi\left(\beta_{i}\left(v_{\|} \cos \theta-v_{g}\right)\right)\right)\left(1+2 \beta_{i}^{2} v_{\|}^{2} \cos ^{2} \theta\right) .
\end{aligned}
$$

The ion flux (7) or (9) and consequently, the current on the collector are the functions of the density, the temperature and the velocity of the different ion species and vary with the potential that is applied to the retarding grids. These parameters can be reconstructed by the fitting of the current, which is measured on the collector, by the model functions (7) or (9).

\subsubsection{Numerical simulation using a Monte-Carlo method}

There exists an effect whose contribution is rather difficult to estimate analytically. This is related to the losses of ions on the side walls of the analyser in the conditions of a non-zero retarding potential. A numerical code using the Monte-Carlo method gives a possibility to quantify this effect and to check the precision of the analytical solutions. The main idea of this method is to generate a large number, $N$, of the test particles with the velocity distribution that corresponds to the expected plasma conditions and then to follow the trajectory of each particle inside of the analyser and to calculate the current that is associated with the particle that arrives at the collector. The position of a test particle on the first diaphragm $g_{1}$, with the section $S_{1}$, is generated randomly and its velocity is chosen as $v_{z}=v_{\|}+G\left(v_{T}\right), v_{x}=v_{x 0}+G\left(v_{T}\right), v_{y}=$ $v_{y 0}+G\left(v_{T}\right)$; here, $G\left(v_{T}\right)$ is the Gaussian probability function with thermal width $v_{T}$, and $\mathbf{v}_{\|}=\left\{v_{x 0}, v_{y 0}, v_{\|}\right\}$is the bulk velocity of the ion population in the satellite frame. The trajectory of each particle is followed from level to level (here we call the "level" either a grid or collector, considered as equipotential planes). For each pair of the neighbouring levels, $g_{k}$ and $g_{k+1}$, the following parameters are calculated:

1. Electric field parallel to $z$-axis, $E_{z}=-\Delta \varphi_{g k} / \Delta z_{k}$; here, $\Delta \varphi_{g k}$ is the potential difference between $k+1$ and $k$ levels separated by the distance $\Delta z_{k}$;

2. Component of the velocity vector that is parallel to $z$-axis on the $(k+1)$ level, $v_{z k+1}=$ $\sqrt{v_{z k}^{2}-[2 k e / m] \Delta \varphi_{g k}}$

3. Fly-time of particle, which is $\Delta t_{k}=\Delta z_{k} / v_{z k}(1-$ $\sqrt{1-\chi}) /[\chi / 2]$, with $\chi=e \Delta \varphi_{g k} /\left[m v_{z k}^{2} / 2 k\right]$;

4. Particle position on the $(k+1)$ level, $x_{k+1}=x_{k}+v_{x} \Delta t_{k}$ and $y_{k+1}=y_{k}+v_{y} \Delta t_{k}$.

If $\chi>1$ then the test particle has not enough energy to overcome the potential barrier and is subsequently lost. The position of the ion that arrives on the $(k+1)$ level is controlled with the aim to verify that it is not lost on the side wall of analyser. Finally, if the particle successfully reaches the collector, its contribution, which is $e S_{1} n_{i} v_{1} / N$, where $v_{1}$ is the magnitude of the particle velocity on the entrance grid $g_{1}$, is added to the total current.

\subsubsection{Comparison of analytical and numerical solutions}

Ions that enter into the analyser are partly absorbed on the side structures and, therefore, not all of them reach the collector. If plasma may be considered as cold and moving along the instrument axis, then the particles will be lost only on the structure which supports the grid $g_{2}$ of APR (see Fig. 1a), but all particles that pass the diaphragm $g_{2}$ will reach the collector and contribute to the current. In such a situation the current on the collector may be calculated by 
using Eq. (2) with the flux given by Eq. (7) and with the entrance area, which is just the open section of the diaphragm $g_{2}$.

What is the contribution of thermal ions? Thermal ions will be retarded by the electric field that is created by the voltage difference between the grids $g_{2}$ and $g_{3}$. If their velocity transversal to the analyser axis is not zero, then they may collide with the wall structure. The probability of such loses depends on the analyser geometry and on the ratio between the thermal and the bulk velocities. Thus, if the transversal displacement of an ion in the layer between grids $g_{2}$ and $g_{3}$ is larger than the difference between the radii of the $g_{3}$ and $g_{2}$ diaphragms, then the ion will be lost. Nevertheless, for the APR geometry and for the expected amplitudes of the thermal and the bulk velocities, the displacement of thermal ions does not exceed the value $2 \Delta z v_{T} / v_{\|}$, with $v_{T}=\sqrt{2 k T / m}$, and thus is not so strong; therefore, most of the thermal particles reach the collector. Consequently, the current produced on the collector by the thermal population may be estimated by using again the cold plasma approximation with the diaphragm area, which ought to be replaced by its effective value, $S_{*}$. The effective area of the entrance diaphragm may be estimated by knowing the ratio of the ion fluxes given by the 1-D and 3-D solutions (Eqs. 7 and 9) and is written in the form $S_{*}=S F_{3-D}\left(v_{g}=0\right) / F_{I D}\left(v_{g}=0\right)$. Figure 2 shows the current-voltage response of the APR analyser to oxygen (a), helium (b) and hydrogen (c), with $T_{i}=0.086 \mathrm{eV}$ and $n_{i}=10^{10} \mathrm{~m}^{-3}$, as computed using the numerical code (black line) and the $1-\mathrm{D}^{*}$ solution for the ion flux with the effective area of the entrance diaphragm, $S^{*}$, (red line). In the considered examples the ratio $S^{*} / S$ is estimated to be $1.01,1.04$, 1.14 for $\mathrm{O}^{+}, \mathrm{He}^{+}, \mathrm{H}^{+}$, respectively. The analytical solution fits well the numerical one for oxygen and helium, but there exists a small discrepancy for hydrogen. This discrepancy comes from the superthermal ions that are partly lost on the side walls. Green lines in the same figures represent the 3$\mathrm{D}$ analytical solution in which the losses of particles are not taken into account.

To demonstrate how well the proposed analytical solution may fit the current-voltage response in the multi-specious plasma, we consider two examples: both of them correspond to the plasma parameters that were found from the IRI model for the day 21.06.04 and the geographical altitude of $45^{\circ}$. The first example is taken at sub-Sun point (12:00 LT), where plasma with the following parameters is expected to be observed, $T_{i}=0.19 \mathrm{eV}, n_{\mathrm{O}+}=3 \cdot 10^{10} \mathrm{~m}^{-3}, n_{\mathrm{He}+}=$ $n_{\mathrm{H}+}=10^{9} \mathrm{~m}^{-3}$ (Fig. 3a), and the second one corresponds to the midnight time $(24: 00 \mathrm{LT})$ with $T_{i}=0.09 \mathrm{eV}, n_{\mathrm{O}+}=$ $1.6 \cdot 10^{10} \mathrm{~m}^{-3}, n_{\mathrm{He}+}=4 \cdot 10^{9} \mathrm{~m}^{-3}, n_{\mathrm{H}+}=3 \cdot 10^{10} \mathrm{~m}^{-3}$ (Fig. 3b). The satellite potential in both cases is assumed to be $0 \mathrm{~V}$ and the bulk velocity equals the satellite speed. The current-voltage response in such conditions was computed using the numerical code (black line) and then was fitted by the $1-\mathrm{D}^{*}$ solution (red line). The precision of the parameters obtained from the fit is the following: $\Delta T_{i} \approx 0.1 \%, \Delta n_{\mathrm{O}+} \approx$ $0.3 \%, \Delta n_{\mathrm{He}+} \approx 3 \%, \Delta n_{\mathrm{H}+} \approx 3 \%, \Delta v_{\|} \approx 20 \mathrm{~ms}^{-1}$ and $\Delta T_{i} \approx 1 \%, \Delta n_{\mathrm{O}+} \approx 1 \%, \Delta n_{\mathrm{He}+} \approx 10 \%, \Delta n_{\mathrm{H}+} \approx 2 \%$,

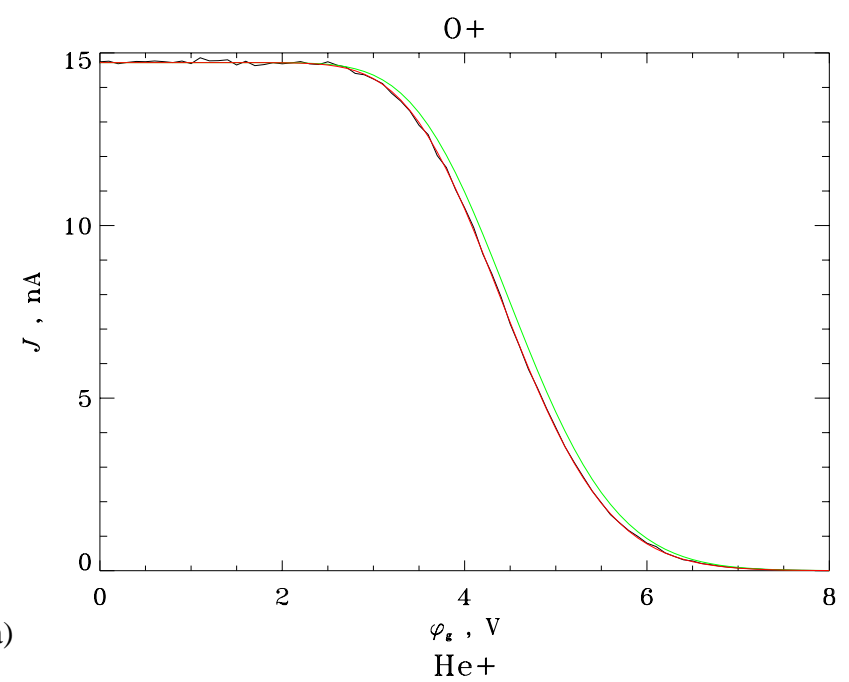

(a)
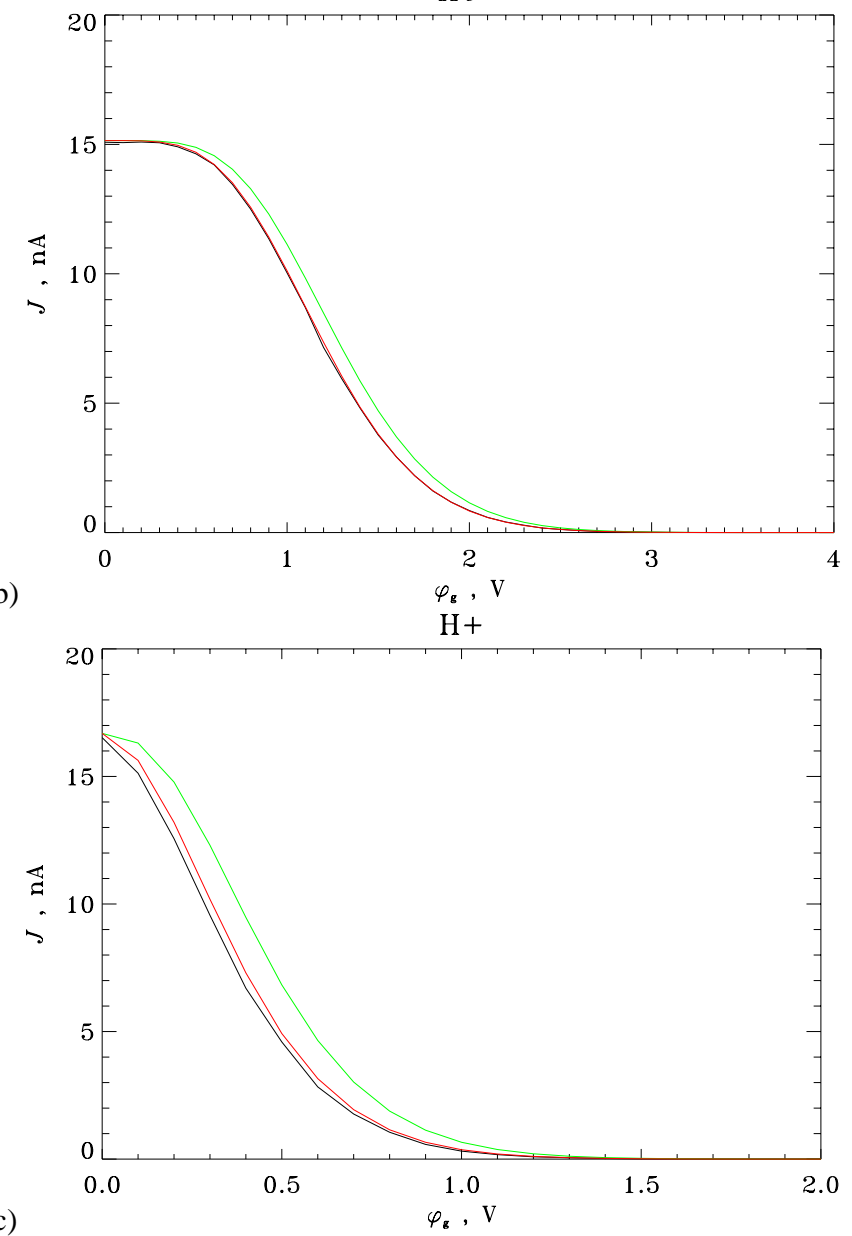

Fig. 2. Current-voltage response of the APR analyser to $\mathrm{O}^{+}$(a), $\mathrm{He}^{+}$(b) and $\mathrm{H}^{+}$(c), with $T_{i}=0.086 \mathrm{eV}$ and $n_{i}=10^{10} \mathrm{~m}^{-3}$, as computed using the numerical code (black line) and the $1-\mathrm{D}^{*}$ solution for the ion flux with the effective section of the entrance diaphragm (red line). Green lines represent a 3-D analytical solution in which the losses of the particles are not taken into account.

$\Delta v_{\|} \approx 50 \mathrm{~m} \mathrm{~s}^{-1}$ for 12:00LT and 24:00 LT, respectively. If the electronic noise with the amplitude at $\sim 10 \%$ of the 


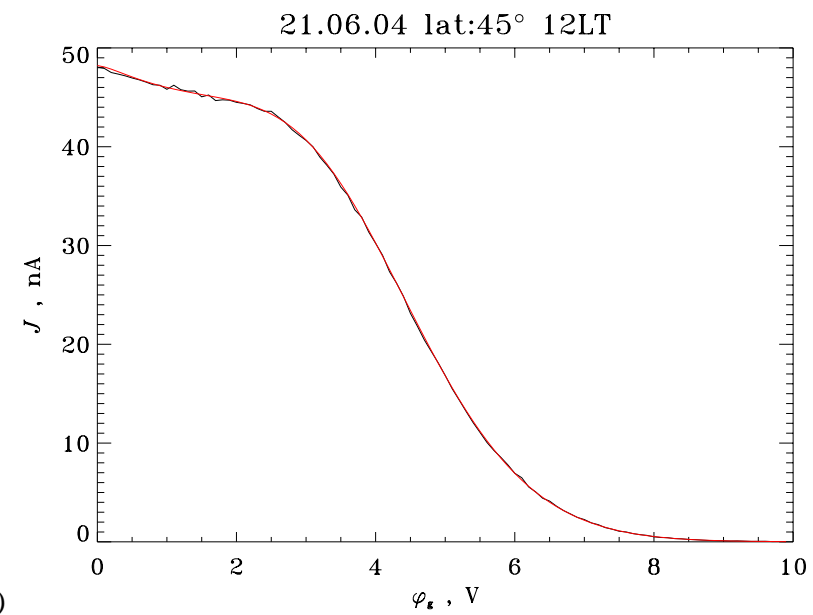

(a)

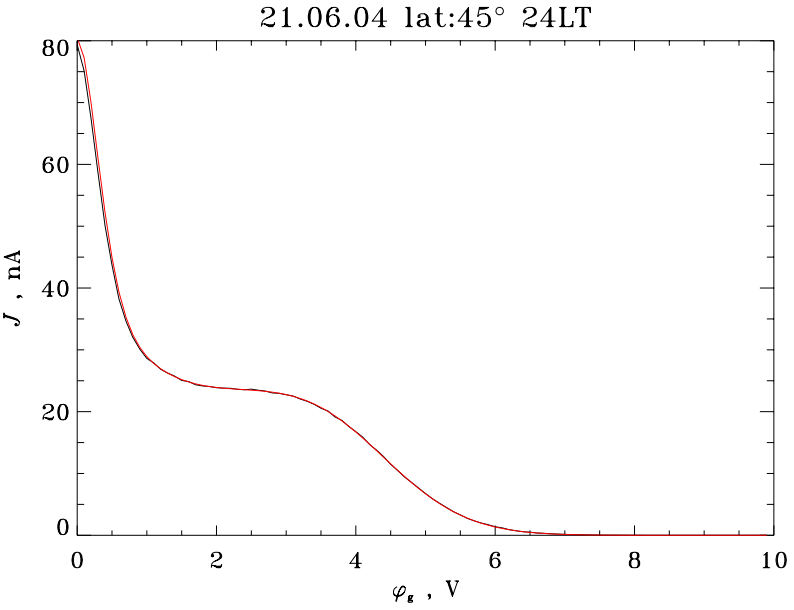

(b)

Fig. 3. Current-voltage response in the multi-species plasma for the conditions, as predicted by the IRI model on 21.06 .04 at the altitude of $45^{\circ}$ : (a) in sub-Sun point (12:00 LT), with $T_{i}=0.19 \mathrm{eV}$, $n_{\mathrm{O}+}=3 \cdot 10^{10} \mathrm{~m}^{-3}, n_{\mathrm{He}+}=n_{\mathrm{H}+}=10^{9} \mathrm{~m}^{-3}$, and (b) at midnight time (24:00 LT) with $T_{i}=0.09 \mathrm{eV}, n_{\mathrm{O}+}=1.6 \cdot 10^{10} \mathrm{~m}^{-3}, n_{\mathrm{He}+}=$ $4 \cdot 10^{9} \mathrm{~m}^{-3} n_{\mathrm{H}+}=3 \cdot 10^{10} \mathrm{~m}^{-3}$. The numerical solution is given by the black line and $1-\mathrm{D}^{*}$ solution by the red line.

collected current is added to the received signal, then the precision is decreased, i.e. $\Delta T_{i} \approx 12 \%, \Delta n_{\mathrm{O}+} \approx 7 \%$, $\Delta n_{\mathrm{He}+} \approx 50 \%, \Delta n_{\mathrm{H}+} \approx 50 \%, \Delta v_{\|} \approx 30 \mathrm{~m} \mathrm{~s}^{-1}$ and $\Delta T_{i} \approx 2 \%, \Delta n_{\mathrm{O}+} \approx 2 \%, \Delta n_{\mathrm{He}+} \approx 20 \%, \Delta n_{\mathrm{H}+} \approx 6 \%$, $\Delta v_{\|}=50 \mathrm{~m} \mathrm{~s}^{-1}$ for 12:00 LT and 24:00 LT, respectively. However, this precision is still sufficient to resolve the main ion components.

\subsection{Deviation of the ion velocity from the analyser axis}

Information about the component of the ion velocity transversal to the analyser axis may be deduced from the ADV measurements. The ADV collector consists of four identical sectors, $a, b, c, d$, which are connected by pairs (Fig. 4). The currents collected by the pairs $a-c, b-d$ and $a-b, c-d$ give a possibility to reconstruct the deviation of the ion bulk velocity from the $z$-axis of the analyser along the axis $x$ and the axis $y$, respectively.
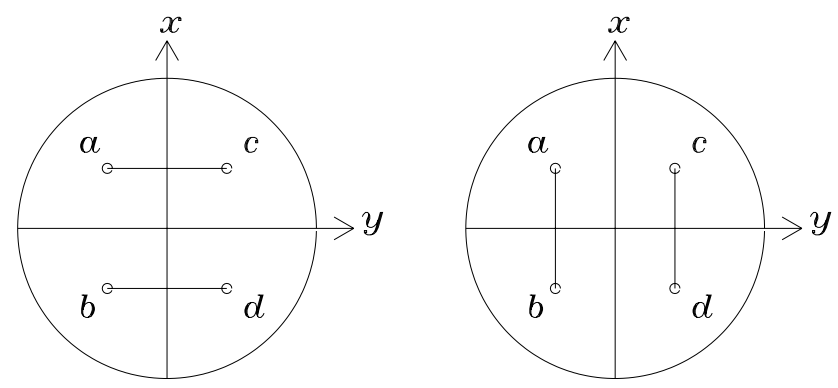

Fig. 4. Four sectors of the ADV collector and their connections in pairs, as it is seen from the entrance diaphragm. The ratios of the currents collected by pairs $a-c$ and $b-d$ (left-hand panel) or $a-b$ and $c-d$ (right-hand panel) give a possibility to derive the deviation of the ion bulk velocity from the analyser's $z$-axis along the $x$ - or $y$-axis, respectively.

First, assume that plasma contains only one ion population. If the thermal velocity of ions, $v_{T}$, is much less than the component of the bulk velocity parallel to the analyser axis, $v_{\|}$, then the ion population may be considered as "cold" and the simple geometrical relation between the ratio of the currents collected by the pairs and the azimuthal, $\varphi$, and the co-latitudinal, $\theta$, angles may be found. It reads

$\alpha_{x}=\left(r_{d}-h \operatorname{tg} \theta \cos \varphi\right) /\left(r_{d}+h \operatorname{tg} \theta \cos \varphi\right)$,

$\alpha_{y}=\left(r_{d}-h t g \theta \sin \varphi\right)\left(r_{d}+h t g \theta \sin \varphi\right)$.

Here, $r_{d}$ is a half-side of the entrance diaphragm $g_{3}$, which has the form of a square, $h$ is the distance between this diaphragm and the collector (Fig. 1b), $\alpha_{x}\left(\alpha_{y}\right)$ is the ratio of the currents measured by the pairs $a-c$ and $b-d$ ( $c-d$ and $a-b), \theta$ is the angle between the ion bulk velocity (in the satellite frame) and $z$-axis, $\varphi$ is the angle in the $x y$ plane. Expressions (12 a, b) are valid when (i) both pairs collect the current and (ii) ion flux that enters in the diaphragm is entirely measured by the collector, i.e. when $\operatorname{tg} \theta<r_{d} / h$ and $\operatorname{tg} \theta<\left(r_{c}-\sqrt{2} r_{d}\right) / h$, where $r_{c}$ is the radius of the collector. For the ADV analyser, with $h=20 \mathrm{~mm}, r_{d}=15 \mathrm{~mm}$ and $r_{c}=$ $35.5 \mathrm{~mm}$, the above mentioned conditions are satisfied when the co-latitudinal angle is less than $35.6^{\circ}$.

In order to justify the limits of the cold plasma approximation, a comparison between the numerical calculations using the Monte-Carlo method and the analytical solution (12) was made. According to the IRI model, there are three major ion species that populate the ionosphere at the altitude of $750 \mathrm{~km}$, i.e. $\mathrm{H}^{+}, \mathrm{He}^{+}$and $\mathrm{O}^{+}$, with a temperature which varies between 0.07 and $0.2 \mathrm{eV}$. If we suppose that the bulk velocity of the particles varies up to $\sim 10 \%$ of the satellite speed, then in the satellite frame the ratio between the thermal to the bulk velocities varies from 0.14 to 0.24 for $\mathrm{O}^{+}$, from 0.28 to 0.47 for $\mathrm{He}^{+}$, from 0.55 to 0.95 for $\mathrm{H}^{+}$. Which ions might be regarded as "cold" and which are not? Figure 5a shows the ratio of the currents collected by the opposite pairs, $a-$ $c$ and $b-d$, of the ADV collector as a function of the colatitudinal angle of the ion flow, given for different values of 
(a)
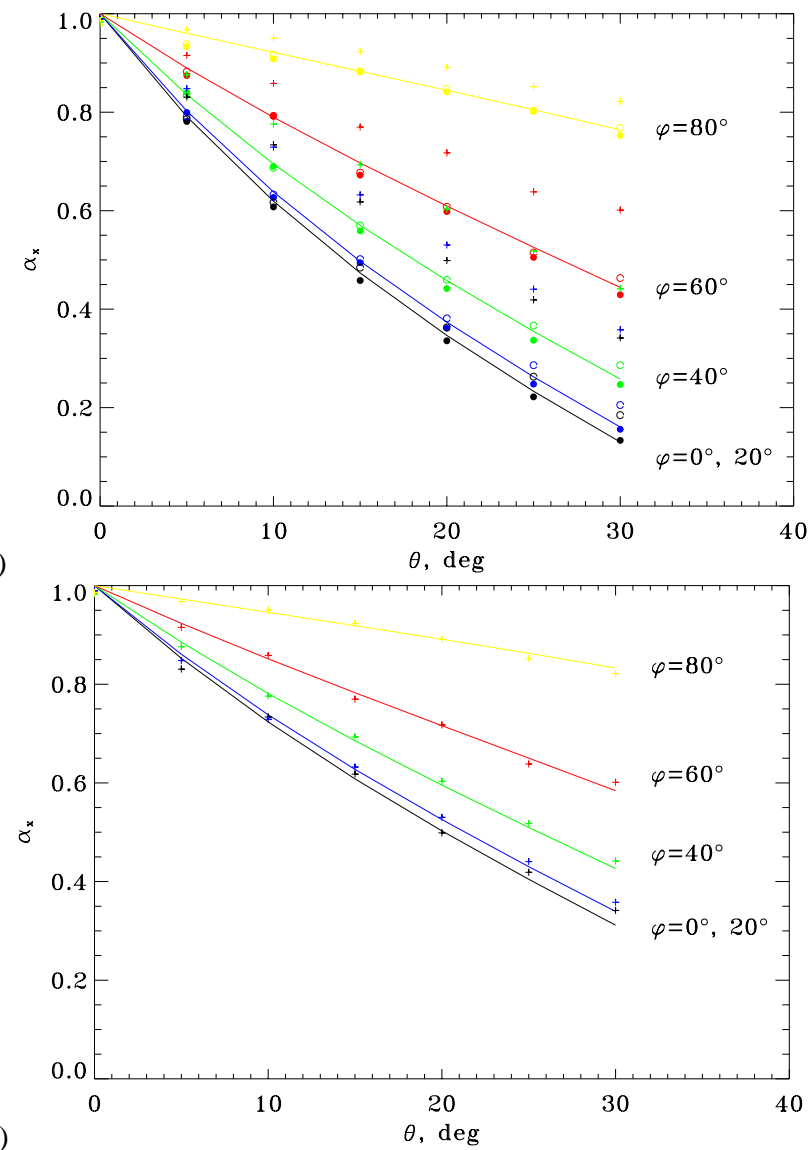

Fig. 5. (a) Ratio of the currents collected by opposite pairs, $a-c$ and $b-d$, of the ADV collector as a function of the co-latitudinal angle of the ion flow, given for different values of the azimuthal angle. The temperature of the ion population is chosen to be $0.2 \mathrm{eV}$ and the parallel velocity is $7.25 \mathrm{~km} \mathrm{~s}^{-1}$. Filled and open circles and crosses represent the results of the numerical calculation for $\mathrm{O}^{+}, \mathrm{He}^{+}, \mathrm{H}^{+}$, respectively. Solid lines show the behaviour which is predicted by the "cold plasma" approximation (12a). Black, blue, green, red and yellow colours are used to plot the analyser's response for the ions flows with the different azimuthal angles, i.e. $0^{\circ}, 20^{\circ}, 40^{\circ}, 60^{\circ}$ and $80^{\circ}$. (b) Current ratio that is produced on the collector by the hydrogen flow (crosses) in the same plasma conditions as in (a). Solid lines show the behaviour that is fitted by Eq. (12a), in which the diaphragm's half-size is replaced by its effective value, $r_{d}^{*}=$ $22 \mathrm{~mm}$.

the azimuthal angle. The temperature of the ion population is chosen to be $0.2 \mathrm{eV}$ and the parallel velocity is $7.25 \mathrm{~km} \mathrm{~s}^{-1}$. Filled and open circles and crosses represent the results of the numerical calculations for oxygen, helium and hydrogen, respectively. Solid lines show the behaviour that is predicted by the "cold plasma" approximation (12a) and, as it is seen from the figure, perfectly fit the points that are attributed to $\mathrm{He}^{+}$and $\mathrm{O}^{+}$(open and filled circles), but not $\mathrm{H}^{+}$(crosses). The poor agreement of hydrogen is because its thermal velocity is a significant fraction of the drift velocity which reduces the current difference between the anode pairs. The current ratio produced on the collector by the hydrogen flow may be

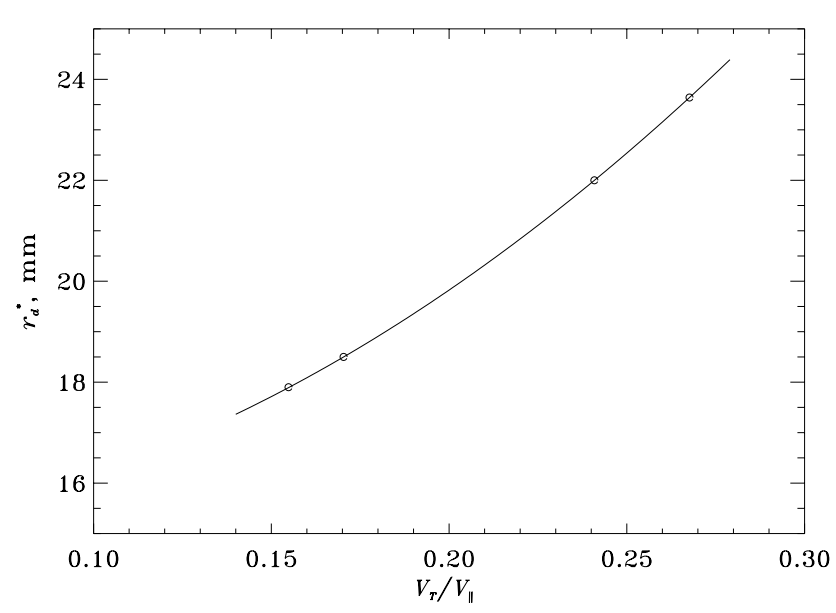

Fig. 6. Effective size of the entrance diaphragm as a function of the ratio between thermal and bulk velocities.

fitted by the expression identical to Eqs. (12a, b), in which the diaphragm half-size, $r_{d}$, is replaced by its effective value, $r_{d}^{*}$. For example, the current responses of the ADV sensor to the hydrogen flows (crosses in the Fig. 5a) are fitted by Eq. (12a) with $r_{d}^{*}=22 \mathrm{~mm}$ (Fig. 5b). The effective size of the diaphragm is a function of the ratio between the thermal and the bulk velocities, and follows the empirical law

$r_{d}^{*}=r_{d}+c\left[V_{T} / V_{\|}\right]^{2}$,

with $c \approx 10$ in the case of the ADV geometry (Fig. 6).

Overall, in plasma with one ion species the measured ratio of currents gives a possibility to reconstruct the arrival angles of the ion flow. The angles may be estimated immediately from Eqs. (12a, b) and reads

$\operatorname{tg} \theta=\frac{r_{d}^{*}}{h}\left[\left[\frac{1-\alpha_{x}}{1+\alpha_{x}}\right]^{2}+\left[\frac{1-\alpha_{y}}{1+\alpha_{y}}\right]^{2}\right]^{0.5}$,
$\cos \varphi=\frac{\alpha_{x}-1}{\alpha_{x}+1}\left[\left[\frac{1-\alpha_{x}}{1+\alpha_{x}}\right]^{2}+\left[\frac{1-\alpha_{y}}{1+\alpha_{y}}\right]^{2}\right]^{-0.5}$,

with $r_{d}^{*}=r_{d}$, if the measured ions are $\mathrm{He}^{+}, \mathrm{O}^{+}$, etc., and with $r_{d}^{*}$, given by Eq. (13), for $\mathrm{H}^{+}$.

Now consider a situation when plasma contains more than one ion population. From the previous considerations it follows that the ratio of the currents that are expected to be collected by the ADV sensor depends only on the arrival angles of ion flows in the plasma without hydrogen. In the presence of hydrogen the measured ratio of currents is determined not only by the arrival angles, but also by the ratio between the thermal and bulk velocities and by the relative concentration of the ion species. In the multi-species plasma the current ratio may be written in the following form

$$
\begin{aligned}
A_{x} & =\frac{(1+\kappa) a_{x} \alpha_{x}^{*}+a_{x}+\kappa \alpha_{x}^{*}}{(1+\kappa)+\kappa a_{x}+\alpha_{x}^{*}}, \\
A_{y} & =\frac{(1+\kappa) a_{y} \alpha_{y}^{*}+a_{y}+\kappa \alpha_{y}^{*}}{(1+\kappa)+\kappa a_{y}+\alpha_{y}^{*}} .
\end{aligned}
$$


Here, $\kappa$ is the relative concentration of hydrogen, $\kappa=$ $n_{H+} / n, \alpha_{x}, \alpha_{y}$ and $\alpha_{x}^{*}, \alpha_{y}^{*}$ are the ratios of the currents that are produced on the collector by the ions with the relative masses $m_{i} / m_{H+} \geq 4$, and by $\mathrm{H}^{+}$, if they are measured separately. The combination of Eqs. (12) and (15) provides the solution for the arrival angles of the ion flow with the assumption that ions move with the same velocity. It reads

$$
\begin{aligned}
& \operatorname{tg} \theta=\frac{(1+\kappa) r_{d} r_{d}^{*}}{\left(\kappa r_{d}+r_{d}^{*}\right) h}\left[\left[\frac{1-A_{x}}{1+A_{x}}\right]^{2}+\left[\frac{1-A_{y}}{1+A_{y}}\right]^{2}\right]^{0.5}, \\
& \cos \varphi=\frac{A_{x}-1}{A_{x}+1}\left[\left[\frac{1-A_{x}}{1+A_{x}}\right]^{2}+\left[\frac{1-A_{y}}{1+A_{y}}\right]^{2}\right]^{-0.5} .
\end{aligned}
$$

However, the last assumption may break down in a collisionfree plasma, because the streaming along the magnetic field may be different for different species and at different energies. This will be true, for example, if there exists a fieldaligned heat flux in the ion population. If this occurs, the arrival angles estimated from the ADV measurements will reflect the convective velocity of the major ion species, generally either oxygen or hydrogen. In order to remove uncertainty in the interpretation of the analyser measurements, the access of hydrogen on the collector may be suppressed by applying the positive potential, $+2 \mathrm{~V}$, to the input grid, $g_{2}$ (Fig. 1b).

\section{Conclusions}

The main idea of the present study was to provide and to justify sufficiently simple analytical tools that may be used to derive the ion flows from the current measurements. It was proven that in the conditions that are expected to be observed during the Demeter mission, i.e. when the bulk plasma velocity in the satellite frame is larger than the ion thermal velocity,

1. The current-voltage response measured by the APR analyser is fitted reasonably well by the 1-D solution in which the contribution of the ion thermal motion transversal to the analyser axis is taken into account by introducing an effective area of the entrance diaphragm;
2. Even a minor ion population may be resolved from the APR response;

3. The current ratio measured by the ADV sensor may be fitted by an expression that arises from the simple geometrical considerations, in which the size of the entrance diaphragm has been replaced by its effective value if the current on the collector is produced by the ions flows with the characteristic thermal velocities which consist of more than half of the bulk speed;

4. The combination of the APR and ADV measurements provides a possibility to reconstruct the arrival angles of the ion flows in the multi-species plasma.

Acknowledgements. This work was supported by the contract 736/CNES/7621. We thank J.-J. Berthelier for useful discussions and acknowledge the engineers of CETP, M. Godefroy, Y. Rennard and F. Leblanc, who developed the IAP instrument.

Topical Editor M. Lester thanks a referee for his help in evaluating this paper.

\section{References}

Hanson, W. B., Sanatani, S., Zuccaro, D., and Flowerday, T. W.: Plasma measurements with the retarding potential analyser on OGO 6, J. Geophys. Res., 75, 5483-5501, 1970.

Hanson, W. B., Frame, D. R., and Midgley, J. E.: Errors in retarding potential analyzers caused by nonuniformity of the grid-plane potential, J. Geophys. Res., 77, 1914-1922, 1972.

Hanson, W. B., Sanatani, S., and Zuccaro, D.: The martian ionosphere as observed by the Viking retarding potential analysers, J. Geophys. Res., 82, 4351-4363, 1977.

Knudsen, W. C.: Evaluation and demonstration of the use of retarding potential analysers for the measuring several ionospheric quantities, J. Geophys. Res., 71, 4669-4678, 1964.

Knudsen, W. C., Spenner, K., Bakke, J., and Novak, V.: Pioneer Venus orbiter planar retarding potential analyser plasma experiment, IEEE Trans. Geos. Rem. Sens., 1, 49-54, 1980.

Whipple, E. C.: The ion trap results, in: "Exploration of the upper atmosphere with the help of the third soviet sputnik", Proc. IRE, 47, 2023-2024, 1959. 